\title{
Mental Images in Body Integrity Identity Disorder (BIID) and in Body Dysmorphic Disorder (BDD): An Exploratory Study
}

\author{
Anja Grocholewski ${ }^{1 \star}$, Carina Bücker ${ }^{1}$, Erich Kasten ${ }^{2}$, Bernhard Osen ${ }^{3}$ and Nina Heinrichs ${ }^{1}$ \\ ${ }^{1}$ Department of Psychology, Braunschweig University, Braunschweig, Germany, ${ }^{2}$ Department of Psychology, \\ Medical School Hamburg, Hamburg, Germany and ${ }^{3}$ Schön Clinic Bad Bramstedt, Bad Bramstedt, Germanhy \\ *Corresponding author. Dr Anja Grocholewski, Clinical Director of the Outpatient Unit for Body Dysmorphic Disorder, \\ Braunschweig University, Department of Psychology, Humboldtstr. 33, 38106 Braunschweig, Germany. \\ Email: anja.grocholewski@tu-braunschweig.de
}

(Received 21 October 2016; revised 18 June 2018; accepted 2 July 2018)

\begin{abstract}
Little is known about mental images (cognitive representations with sensory qualities) in body integrity identity disorder (BIID). This study aimed to determine whether individuals with BIID experience mental images and, if so, whether they differ to those experienced by individuals with body dysmorphic disorder (BDD) and healthy controls. A semi-structured interview on mental images and their qualities was delivered to a sample of 22 individuals with BIID and 19 with BDD, and 22 healthy controls. Both the BIID $(n=15,68.2 \%)$ and BDD $(n=14,73.7 \%)$ groups reported significantly more mental images than healthy controls $(n=3,13.6 \%)$, but no differences between the two clinical groups were found. While mental images in BDD were focused on 'flawed' body parts, images in BIID involved the desired handicap. Mental images in BIID were experienced as less distressing and more sexually arousing compared to BDD. No further differences were found between the groups (e.g., regarding sensory multimodality, dominance of a specific body part). Although the two groups reported a similar frequency of mental images, images appear to serve different functions across conditions. Clinically, it may be necessary to target the positive mental images in BIID to improve treatment outcome.
\end{abstract}

Keywords: mental images; body integrity identity disorder; body dysmorphic disorder

In the last decades, interest in the experience of mental images has been growing - for example in research relating to anxiety disorder, post-traumatic stress disorder (PTSD), body dysmorphic disorder (BDD), or eating disorders (Brewin, Gregory, Lipton, \& Burgess, 2010; Onden Lim, 2013). Mental images are cognitive representations that are not purely verbal or abstract, but possess sensory qualities (Horowitz, 1970) and can provoke experiences of 'seeing with the mind's eye' (Kosslyn, Ganis, \& Thompson, 2001). The emotional valence of an image can be rated positive, neutral, or negative (Hackmann, Surawy, \& Clark, 1998). They can be activated by external stimuli and subjectively experienced as real and intrusive or uncontrollable (Hackmann, Bennett-Levy, \& Holmes, 2012). Holmes and Mathews (2010) described how mental images are not only created by visual images in the mind and perceptual impressions (e.g., the impression of smelling something), but can also be linked to autobiographical memories. Moreover, these images are not limited to visual representations (sight), but can also include other sensory modalities such as olfactory (smell), auditory (sound), gustatory (taste), and haptic or tactile (touch) impressions (Hackmann et al., 2012; Horowitz, 1967; Kosslyn et al., 2001; Onden Lim, 2013).

The large variety of modalities of images also involves an interaction between cognitions, emotions, memory and perception, and contributes to the impression that mental images may be experienced as 
particularly lively but also as stressful (Brewin et al., 2010). Furthermore, it is possible that mental images impair the distinction of imagined and real content (Holmes \& Mathews, 2010). For example, in individuals with social anxiety disorder or PTSD, the memories of negative situations and related mental images can lead to maladaptive behaviour (Hackmann, Clark, \& McManus, 2000; Hirsch, Clark, Mathews, \& Williams, 2003; Holmes, Arntz, \& Smucker, 2007a). Moreover, Holmes, Crane, Fennell, and Williams (2007b) reported that the perceived realness of mental imagery of suicide seems to be associated with the severity of suicidal ideation (Holmes, Crane, Fennel, \& Williams, 2007b). Mental images may therefore trigger or reinforce dysfunctional behaviour (e.g., self-harming behaviour).

The great potential of mental images to trigger strong emotional states may have led to their use as a direct target in treatment approaches (Holmes et al., 2007a). Using 'imagery rescripting', individuals with social anxiety disorder, PTSD or BDD can learn to modify their images and associated emotions (Holmes et al., 2007a; Ritter \& Stangier, 2016; Wild, Hackmann \& Clark, 2008; Willson, Veale, \& Freeston, 2016). In sum, recurrent mental images that illustrate negative events are often associated with negative affective experiences and inappropriate behaviour changes in emotional disorders (Holmes \& Mathews, 2010). It is unclear whether these associations are unique to emotional disorders (such as anxiety disorders or PTSD), or whether they are also relevant for individuals with other conditions.

\section{Body Integrity Identity Disorder (BIID) and Mental Images}

Individuals with BIID feel that healthy parts of their body (e.g., a leg or an arm) do not belong to their own body, and some feel a strong need for an amputation of the limb (First, 2005; Oddo, Möller, \& Stirn, 2014; Stirn, Thiel, \& Oddo, 2010). Other individuals affected seek to be paraplegic, blind or deaf (First \& Fisher, 2012; Giummarra, Bradshaw, Hilti, Nicholls, \& Brugger, 2012). In rare cases, affected individuals undertake life-threatening self-amputation experiments (First, 2005; Sorene, Heras-Palou, \& Burke, 2006; Stirn et al., 2010); they feel that only the body modification (i.e., amputation of an intact body part) would help them to feel 'complete'. Unlike BDD, individuals with BIID have good insight that their desires are 'unusual' and not socially desirable (Stirn et al., 2010). The few available models on the aetiology of this condition are mainly neurological or psychological/psychiatric (Sedda \& Bottini, 2014). However, there is little overlap between methods used (e.g., imaging methods like fMRI, psychophysics, or physiological measures) across relevant studies (Sedda \& Bottini, 2014). Moreover, many studies have focused on individuals with the desire for amputation of a lower limb. These results may not be suitable for individuals with the desire for amputation of upper limbs, or with the desire to be paraplegic, blind or deaf (Sedda \& Bottini, 2014). The primary neurological explanation refers to a parietal lobe dysfunction (Sedda \& Bottini, 2014). Psychological models assume a key event in early childhood (e.g., meeting an amputated war veteran) that leads to a spontaneous fascination for amputees (Stirn et al., 2010). Obernolte, Schnell, and Kasten (2015) found that individuals with BIID remembered more childhood experiences with disabled or severely physically ill people than individuals without BIID, but it is unclear whether they had more contact with disabled people, or just had better memories of the encounters (Obernote et al., 2015). BIID sufferers also recalled admiring individuals with amputations or palsy in their childhood (Obernolte et al., 2015). Stirn et al. (2010) suspect that in the beginning, the playful, imaginative desire for disability might indicate positively perceived mental images. During puberty, sexual excitement about these positive images may turn them into a desire to have the disability (Stirn et al., 2010). Indeed some BIID participants report sexual motives for amputation (First, 2005; Oddo et al., 2014; Stirn et al., 2010), indicating these images are sexually arousing for a subsample (First, 2005; Kasten, 2009). However, to the best of our knowledge, no study has specifically explored the 'nature' of mental images in individuals with BIID. Holmes and colleagues (2007a) argued that identifying the occurrence, frequency, and functions of mental images may help to develop additional treatment modules. Furthermore, comparing the descriptive nature of mental images across conditions may help to identify condition-specific characteristics. This approach might facilitate the identification of different (or similar) functions, which in turn may offer important targets for psychological change. 


\section{Body Dysmorphic Disorder and Mental Images}

The primary indicator of BDD is the excessive preoccupation with an appearance-related defect, which is exaggerated in its severity (American Psychiatric Association [APA], 2013). BDD is often associated with obsessions that can become delusional. For example, Phillips (2004) found 84\% of BDD patients $(n=129)$ had either delusional obsessions $(n=68)$ or poor insight that their appearance beliefs were exaggerated $(n=40)$. Moreover, individuals with BDD have a tendency to body modification such as plastic surgery. Two population-based surveys in Germany found that 7.2-15.6\% of participants with BDD underwent cosmetic surgery compared to $2.8-3.0 \%$ of individuals without BDD (Buhlmann et al., 2010; Rief, Buhlmann, Wilhelm, Borkenhagen, \& Brähler, 2006). The importance of mental images in BDD was examined in several studies (e.g., Onden Lim, 2013; Osman, Cooper, Hackmann, \& Veale, 2004). People with BDD tend to focus their attention on their appearance, particularly their disfigured body parts (Neziroglu, Khemlani-Patel, \& Veale, 2008). It is assumed that this attentional bias reinforces distorted mental images (Veale, 2004). These mental images are triggered by external events (e.g., looking in a mirror), as well as intrusive thoughts (e.g., thinking about their ugly appearance; Veale, 2004). In the study by Osman et al. (2004), all 18 participants with BDD reported experiencing either mental images or other sensory impressions. Interestingly, 15 (out of 18) individuals without any mental disorder also reported experiencing either mental images or other sensory impressions. Although the two groups did not differ in the frequency of mental images, individuals with BDD reported more vivid, intense and negatively valenced images (Osman et al., 2004). Moreover, individuals with BDD reported seeing images through the eyes of another individual rather than their own, otherwise known as the observer perspective (Onden Lim, 2013; Osman et al., 2004). This observer-taking perspective has also been reported in studies on mental images in social phobia (Hackmann et al., 1998). Veale (2004) reports that the observer perspective allows individuals with $\mathrm{BDD}$ enough distance from their images to avoid experiencing negative feelings about them. Such emotional avoidance may become a maintaining factor for BDD (Veale, 2004).

\section{Aims of This Study}

We wanted to explore whether individuals with BIID: (1) actually experience mental images, and, if so, (2) to what extent these images differ from (or overlap with) the mental images of individuals with $\mathrm{BDD}$ and mentally healthy controls. BDD was selected as the clinical comparison group due to the similarities of this condition with BIID, in terms of the presence of body representation disturbance and the desire ('ideation') for body modification (e.g., amputation in BIID, plastic surgery in BDD). This study aimed to compare the frequency, sensory modality, link to autobiographical memories, and dominance of a body part in the mental images of people with BIID, BDD, and healthy controls. We hypothesised that both clinical groups would experience more frequent mental images, report a broader range of sensory perceptions, produce more autobiographical memories, and be more likely to focus on a specific body part within their mental images than mentally healthy controls, but would not differ from each other in these variables. The mental images of the BIID and BDD groups were then compared on the level of concern, distress, sexual arousal, intrusiveness and insight into their mental images, as well as the perspective from which they are seen. Individuals with BIID were expected to report less distress from their mental images than individuals with BDD, due to their focus on idealised body representations (e.g., the desired body; Sorene et al., 2006), rather than the flawed body representations seen in BDD, which is more distressing (Veale, 2004). BDD and BIID are characterised as disorders with low versus high levels of insight respectively (Phillips, 2004; Stirn et al., 2010). In this study, we expected the two groups to differ in rational (the knowledge that an image may be distorted) but not emotional (the feeling that images are real) insight, given the severe suffering (First, 2005; Sorene et al., 2006; Stirn et al., 2010; Veale, 2004) and high degree of identification with the mental image in both clinical conditions. We also hypothesised that individuals with BIID would report more sexually arousing and less intrusive mental images. In contrast to 
$\mathrm{BDD}$, individuals with BIID produce mental images more intentionally, due to the rewarding nature of the desired disability (Stirn et al., 2010). Several studies reported that BIID participants expressed sexual motives for the desired amputation (First, 2005; Oddo et al., 2014; Stirn et al., 2010). Given this finding, we hypothesised that the BIID group in this study would report more sexual arousal associated with their mental images compared to the BDD group. Finally, we expected the BDD group would take more of an observer perspective of their images than the BIID group, because individuals with BDD want more distance from their distressing images than those with BIID, who want more closeness to their images (Veale, 2004).

\section{Methods}

\section{Participants and Sampling Procedure}

Participants with BIID were recruited from an online self-help forum and specialised outpatient units or private practices. The desired disabilities of the BIID participants are shown in Table 1. BDD participants were recruited from two settings: a university outpatient unit and a psychosomatic inpatient unit. Eleven of the BDD participants were assessed in the context of a master's thesis (concerning mental images in BDD and social anxiety disorder; Rösner, 2014). Mentally healthy controls were recruited from the community via word-of-mouth advertising. Exclusion criteria for the clinical groups were: (1) comorbid disorders that may have an influence on the incidence of mental images (e.g., social anxiety disorder, PTSD, alcohol or drug abuse, dementia, or psychotic disorders) and (2) simultaneously meeting diagnostic criteria for both BIID and BDD. Participants of the control group were not allowed to meet criteria for any current mental disorder. For all individuals, participation was voluntary and commenced after informed consent had been provided. The study was reviewed by an Ethical Review Board prior to study commencement. The Mini-DIPS (Margraf, 1994) was conducted to screen for mental disorders in the control group. To assess presence of BIID symptoms, the screening questionnaire by Stirn et al. (2010) was used. To check eligibility and assess inclusion criteria for the BDD group, we used the German version of the Body Dysmorphic Disorder Diagnostic Module (BDDDM; Phillips, 1986; Stangier, Hungerbühler, \& Meyer, 1996), in addition to the BDD diagnosis already assigned by therapists in the university outpatient unit and the psychosomatic inpatient unit.

In total, 66 individuals were assessed. Three subjects were excluded (two who were assumed to meet criteria for psychosis and one who met criteria for both conditions). The total sample consisted of 19 BDD, 22 BIID, and 22 control participants $(N=63)$. The sample was recruited between January and May 2015 (active recruitment ended after a sample size of 20 per group had been reached, with one BDD individual subsequently excluded). As can be seen in Table 2, all three groups differed in age, gender, and marital status. The BIID group were significantly older, more likely to be male, and married. There were no differences in education or employment. The implications of these differences are discussed in the study limitations.

\section{Assessments}

\section{Mental images interview}

We assessed mental images with an adapted version of the semi-structured interview by Osman et al. (2004), who used the first version developed by Hackmann et al. (1998) for anxiety disorders and adapted it to the context of individuals with BDD. In this interview, participants are requested to think about a recent time when they have felt worried about their appearance. They are asked whether they have ever experienced any spontaneous images (or if not, whether they have experienced an impression of the way they think they look). They are also asked whether the images and/or impressions are recurrent, such that they always involve the same things (Osman et al., 2004). For one of the images, participants are asked if seven sensory modalities (visual, auditory, cutaneous, kinaesthetic, 
Table 1. Desired Disabilities of the $N=22$ BIID-Participants

\begin{tabular}{ll}
\hline Desired disabilities & $n(\%)$ \\
\hline (Incomplete) paraplegia & $4(18.2 \%)$ \\
\hline Paralysis both legs & $1(4.5 \%)$ \\
\hline Numbness of both legs & $1(4.5 \%)$ \\
\hline Catheterizing of bladder & $1(4.5 \%)$ \\
\hline Amputation left leg & $3(13.6 \%)$ \\
\hline Amputation right leg & $4(18.2 \%)$ \\
\hline Amputation both legs & $2(9.1 \%)$ \\
\hline Amputation right arm and right leg & $1(4.5 \%)$ \\
\hline Amputation left arm & $1(4.5 \%)$ \\
\hline Amputation left food & $1(4.5 \%)$ \\
\hline Removal of all fingernails and amputation of left thumb & $1(4.5 \%)$ \\
\hline Deformation (club foot) both feet & $1(4.5 \%)$ \\
\hline Severe walking impairment & $1(4.5 \%)$ \\
\hline
\end{tabular}

organic, gustatory, and olfactory) are present (Osman et al., 2004). To rate the presence of the sensory modalities, participants were asked, for example, whether they can see or hear anything in the image, and had to answer with 'yes' or 'no' (Osman et al., 2004). They are also asked what perspective the image is seen from, rated on a 7-point scale (+3 (observer perspective) to -3 (participant perspective) and to rate their level of rational and emotional insight that the content of the mental image is distorted on a $0-100$ scale $(0=$ not at all distorted, $100=$ completely distorted; Osman et al., 2004).

We translated the BDD version into German and adapted this version to the context of our study. First, we broadened the questions: Participants were asked to think about a recent time when they had felt worried about their appearance and their body (this was useful for BIID participants, who are typically not concerned about their appearance, but about their body). Furthermore, we added some interview questions to assess information related to our hypothesis. To assess the dominance of specific body parts, we added the question: 'Are there specific body parts in the image that are emphasised?' (answered with 'yes' or 'no'; participants were requested to list these specific body parts after the item). To investigate the hypothesis regarding concern and distress, we used the item from the original semi-structured interview, which asks whether the mental image is perceived as a cause for concern. To assess distress, we added a further item asking about distress when viewing the image, rated on a scale from 1 to $100(0=$ no distress, $100=$ extremely distressing $)$. To detect sexual arousal due to the mental image, the participants were asked whether they are sexually aroused by the mental image ('yes' or 'no'). Due to our interest in the intrusiveness of mental images, we added the item 'Are they intrusive in that they just pop into your mind?', which is part of the mental images interview for BDD recommended by Veale and Neziroglu (2010).

\section{Mini-DIPS}

This structured interview is a brief version of the Diagnostic Interview for Mental Disorders (DIPS; Margraf, Schneider, \& Ehlers, 1991). It is comparable to the Anxiety Disorders Interview Schedule by Brown, Barlow, and DiNardo (2004), but limited to information needed for validating diagnoses. The Mini-DIPS provides an overall assessment of the most important mental disorders according to Diagnostic and Statistical Manual of Mental Disorders (3rd ed., rev.; DSM-III-R; APA, 1987) criteria. Even though the Mini-DIPS is based on DSM-III-R criteria, it is among the very few validated briefinterview screening for mental disorders in a research setting available in German language. The 
Table 2. Socio-Demographic Characteristics

\begin{tabular}{|c|c|c|c|c|c|c|c|}
\hline \multirow{2}{*}{ Variables } & \multicolumn{2}{|c|}{$\begin{array}{c}\text { BDD } \\
(N=19)\end{array}$} & \multicolumn{2}{|c|}{$\begin{array}{c}\text { BIID } \\
(N=22)\end{array}$} & \multicolumn{2}{|c|}{$\begin{array}{l}\text { Controls } \\
(N=22)\end{array}$} & \multirow[b]{2}{*}{$\mathrm{H}$} \\
\hline & $M$ & $S D$ & $M$ & $S D$ & $M$ & $S D$ & \\
\hline \multirow[t]{2}{*}{ Age } & 35.26 & 12.76 & 50.73 & 11.24 & 38.95 & 15.87 & $13.709^{\star *}$ \\
\hline & $N$ & $\%$ & $N$ & $\%$ & $N$ & $\%$ & $\chi^{2}$ \\
\hline \multicolumn{8}{|l|}{ Gender } \\
\hline Male & 6 & 31.6 & 19 & 86.4 & 10 & 45.5 & $13.790^{\star \star}$ \\
\hline Female & 13 & 68.4 & 3 & 13.6 & 12 & 54.5 & \\
\hline \multicolumn{8}{|l|}{ Marital status } \\
\hline Single & 10 & 62.5 & 5 & 22.7 & 5 & 22.7 & $8.352^{*}$ \\
\hline Married & 1 & 6.3 & 6 & 27.3 & 10 & 45.5 & $7.031^{\star}$ \\
\hline Divorced & 1 & 6.3 & 4 & 18.2 & 0 & 0 & 4.884 \\
\hline Partnership & 3 & 18.8 & 7 & 31.8 & 7 & 31.8 & .987 \\
\hline Widowed & 1 & 6.3 & 0 & 0 & 0 & 0 & 2.797 \\
\hline \multicolumn{8}{|l|}{ Education } \\
\hline 9 years & 3 & 21.4 & 1 & 4.5 & 2 & 9.1 & 2.689 \\
\hline 11 years & 4 & 28.6 & 3 & 13.6 & 4 & 18.2 & 1.256 \\
\hline 12 years & 1 & 7.1 & 0 & 0 & 1 & 4.5 & 1.439 \\
\hline High school (13 years) & 4 & 28.6 & 8 & 36.4 & 7 & 31.8 & .250 \\
\hline College/University & 2 & 14.3 & 10 & 45.5 & 8 & 36.4 & 3.736 \\
\hline Employment & 8 & 57.1 & 13 & 59.1 & 15 & 68.2 & .576 \\
\hline
\end{tabular}

Note: $\mathrm{BDD}=$ body dysmorphic disorder; $\mathrm{BIID}=$ body integrity identity disorder; $M=$ mean; $S D=$ standard deviation; $N=$ number of participants; $\%=$ percent; $\mathrm{H}=$ the Kruskall-Wallis test statistics; $\chi^{2}=$ the chi-square test statistics.

${ }^{\star} p<.05 ;{ }^{\star \star} p<.01$

primary conditions in this study (BIID and BDD) were not assessed by the Mini-DIPS, but by other instruments based on DSM-IV-R (APA, 2000) criteria. In the Mini-DIPS, kappa coefficients for $D S M-I I I-R$ categories vary between .76 (somatoform disorders) and 1.0 (psychotic disorders; Margraf, 1994). If isolated criteria for any current mental disorders were met, we compared them with DSM-5 criteria. No current mental disorders were detected in the control group.

\section{BIID screening}

This tool was developed by Stirn et al. (2010). It includes 15 items assessing the desire for disability, suffering, and contact with other BIID sufferers. Items are answered using a 'yes' or 'no' format. Examples of items are 'Have you got the desire for being disabled?', 'Do you pretend in a regular way?', and 'Have you been fascinated by disabled people in your childhood?'. The cut-off score to detect BIID is 8 (number of 'yes' answers).

\section{$B D D D M$}

The Body Dysmorphic Disorder Diagnostic Module (BDDDM, Phillips, 1986) provides information on the presence of BDD. The disorder is diagnosed if all DSM-IV (APA, 1994) criteria for BDD are met (Phillips, 1986). We used the validated German version of the BDDDM (Stangier et al., 1996). 


\section{Data Analyses}

For the calculation of distributions and hypotheses, SPSS Statistics 22.0 was used. To test the stochastic independence of categorical variables, chi-squared tests and odds ratios were calculated. If criteria for conducting chi-squared tests were not met (exclusive assignment of each participant to one category; expected frequency $<5$ ), we used Fisher's exact test (Bortz \& Lienert, 2008). Due to the lack of normal distribution and homogeneity of variance, as expected with small samples, we used non-parametric statistical tests for continuous variables.

\section{Results}

\section{Group Comparison of Individuals With BDD and BIID to Healthy Controls}

\section{Occurrence of a mental image}

Fourteen (out of 19) participants (73.7\%) with BDD experienced mental images compared to 15 (out of 22) participants with BIID (68.2\%). In the healthy control group, 3 out 22 individuals (13.6\%) reported mental images in the interview. The two clinical groups reported experiencing significantly more frequent mental images than controls (BIID - control: $\chi^{2}=13.54, p<.001, d f=1, N=44$, $\omega=.555$; BDD - control $\left.\chi^{2}=15.15, p<.001, d f=1, N=41, \omega=.608\right)$ with large effect sizes. No significant differences in the frequency of mental images was found between the BIID and BDD groups $\left(\chi^{2}=.149, p=.699, d f=1, N=41\right)$.

As a result of the small number of individuals in the control group reporting mental images, the other statistical comparisons between the two clinical groups and healthy controls on sensory multimodality, autobiographical memories, and dominance of a specific body part in mental image were only conducted between the BDD and the BIID groups.

\section{Sensory multimodality of mental images}

When focusing on the number of modalities involved in the mental image (if an image was reported), we found a median of 2.00 modalities in $\operatorname{BDD}(n=14$, minimum $=1.00$, maximum $=5.00)$ as well as in BIID $(n=15$, minimum $=1.00$, maximum $=5.00)$. Participants from the control group reported a median of $1(n=3$, minimum $=1.00$, maximum $x=2: 00)$. The Mann-Whitney $\mathrm{U}$ test between the BDD and BIID groups $(N=29, z=-.500, p=.652)$ was not significant.

\section{Mental image and autobiographical memories}

Under the condition that a mental image existed, the data showed that 8 (out of 14) participants with BDD (57.1\%), 9 (out of 15) participants with BIID (60.0\%) and 2 (out of 3) of the control subjects $(66.7 \%)$ reported mental images that were linked to autobiographical memories. The Fisher's exact test between the BDD and the BIID group $(p>.99)$ was not significant; the odds ratio (no autobiographical memory/autobiographical memory) for BDD was 1.063 , and for BIID it was 0.944 .

\section{Dominance of a body part in the mental image}

Under the condition that a mental image was present, 12 (out of 14) participants with BDD (85.7\%), 13 (out of 15) participants with BIID (86.7\%) and 2 (out of 3) of control participants $(66.7 \%)$ reported mental images involving a dominant specific part of the body. The Fisher-Yates test between the BDD and BIID groups $(p>.99)$ again showed no significant difference. Odds ratio (no dominance of a body part/dominance of a body part) was 1.042 for BDD, and 0.962 for BIID. 
Table 3. Distribution of Concern Distress and Experience of Rational and Emotional Insight Into the Mental Image Distortion

\begin{tabular}{ccccccc}
\hline Variables & $N$ & $M$ & SD & Mdn & Min & Max \\
\hline Concern and distress & & & & & & \\
\hline BDD & 13 & 87.31 & 12.85 & 90.00 & 55.00 & 100.00 \\
\hline BIID & 13 & 27.54 & 41.58 & 0.00 & 0.00 & 89.00 \\
\hline Insight (rational) & 13 & 48.08 & 38.38 & 50.00 & 0.00 & 100.00 \\
\hline BDD & 14 & 52.50 & 40.04 & 60.00 & 0.00 & 100.00 \\
\hline BIID & & & & & \\
\hline Insight (emotional) & 13 & 35.00 & 36.97 & 30.00 & 0.00 & 100.00 \\
\hline BDD & 14 & 14.79 & 21.62 & 0.00 & 0.00 & 60.00 \\
\hline BIID & 13 & & & & \\
\hline
\end{tabular}

Note: $\mathrm{BDD}=$ body dysmorphic disorder; $\mathrm{BIID}=$ body integrity identity disorder; $N=$ number; $M=$ mean; $S D=$ standard deviation; $M d n=$ median; Min $=$ minimum; $\operatorname{Max}=$ maximum; Concern and distress = concern and distress related to the experience of the mental image; Insight (rational) = rational insight into the distortion of the mental image; Insight (emotional) = emotional insight into the distortion of the mental image.

\section{Group Comparison of Individuals With BDD and BIID Who Experience Mental Images}

Concern and distress related to the experience of the mental image

Table 3 illustrates that BDD participants more often experienced concern and distress related to their mental image than BIID participants $\left(\chi^{2}=14.58, p<.001, d f=1, N=28\right)$. This difference was large $(\omega=.722)$. In fact, individuals with BIID were not distressed by the image at all $(M d n=0.00$ on a scale from 0-100), while individuals with BDD had a median of $90.00(z=-2.964, p=.002, N=$ $26)$, and the effect size again showed a large difference $(\varphi=.71)$.

\section{Sexual arousal due to the mental image}

None of the five BDD participants, but $60 \%(n=9)$ of the BIID participants reporting mental images indicated they perceived it to be sexually arousing. The Fisher's exact test was significant, with $p=.04$. The odds ratio (no sexual arousal/sexual arousal) for BIID was 0.545 .

\section{Intrusiveness of the mental image}

The majority of both BDD and BIID subjects reported intrusive mental images (BDD $=83.3 \%, n=10$; $\mathrm{BIID}=72.7 \%, n=8$ ). The Fisher's exact test was not significant, with $p=.640$, with an odds ratio (intrusive/not intrusive) of 1.389 for BDD, and 0.741 for BIID.

\section{Insight into the distortion of the mental image}

In terms of rational insight, BIID subjects $(n=14)$ showed a 60.00 and BDD subjects $(n=13)$ a median $=50.00$ (scale from 0 to 100$)$. The $\mathrm{U}$ test $(z=-.196, p=.434, N=27)$ was not significant.

In terms of emotional insight, BIID subjects $(n=14)$ showed a median of 0.00 and BDD subjects $(n=14)$ showed a median of 30.00 . The $\mathrm{U}$ test $(z=-1.774, p=.094, N=28)$ was not significant.

\section{Perspective from which the mental image was seen}

Here, only data of the participants who described a unique perspective were analysed - data of participants who answered with zero (reflecting a neutral perspective) were excluded. Exploratory analysis showed that BDD subjects reported roughly equal frequencies of participant $(45.5 \%, n=5)$ and observer perspectives $(54.5 \%, n=6)$, while in BIID, the participant perspective (seeing the image with the own eyes) was predominant (participant perspective: $83.3 \%, n=10$; observer perspective: $16.7 \%, n=2)$. The Fisher's exact test failed to reach statistical significance, with $p=.089$, with an odds ratio (participant perspective/observer perspective) of 0.444 for $\mathrm{BDD}$, and 2.667 for BIID. 


\section{Discussion}

The first aim of this study was to compare the mental images of two groups of conditions involving body-related concerns with a control group of mentally healthy individuals. The results showed that the two clinical groups experienced significantly more mental images than the control group. Further differences (in mental images, autobiographical memories, sensory multimodality or dominance of a specific body part) could not be analysed due to the small number of control subjects who experienced mental images.

The second aim was to explore condition-specific characteristics of mental images in participants with BIID and BDD. The two clinical groups did not show statistically significant differences in the majority of the characteristics of the mental images investigated (intrusiveness of the mental image, insight, and perspective from which the mental image was seen). However, they did differ in the distress level associated with experiencing these mental images and their perception as sexually arousing, indicating that individuals with BIID associate more positive emotions with (negative) mental images.

There are a number of conclusions that can be drawn from the study: (1) mental images play a larger role within the two clinical groups than in healthy control subjects; (2) mental images may be similar in quality across both clinical groups, indicating a number of condition-overarching characteristics (cave: sample size); (3) individuals with BDD experience more distress from their mental images. Furthermore, $60 \%$ of the BIID group reported experiencing sexual arousal (interpreted as a positive emotional state) in response to a mental image, compared to no participants in the BDD group. This is consistent with previous studies on BIID, which also reported sexual arousal to be a condition-specific phenomenon in BIID (e.g., First, 2005; Kasten, 2009). Although it has been suggested that these individuals may represent a specific subtype of BIID, there are currently too few empirical studies to support this hypothesis.

In addition, higher levels of rational insight into the distortion of the mental images was expected in the BIID group (First, 2005; Sorene et al., 2006; Stirn et al., 2010; Veale, 2004). This hypothesis was based on clinical observation rather than empirical evidence. Our study clearly showed that both rational and emotional insight in BIID was lower than postulated: in fact, individuals with both BIID and BDD reported low levels of rational insight. Insight into distortions of mental images may not be a discriminating characteristic of these two conditions.

Similarly, no significant differences $(p=.89)$ were found between the perspective taken within the mental image: seeing it as with one's own eyes, or as through the eyes of another individual. Nevertheless, almost equal frequencies of individuals in the BDD group took a participant or observer perspective, while $83 \%$ of those in the BIID group took the participant perspective. While speculative due to the lack of significant results small sample size, and this suggests that the perspective taken within the mental image could be a discriminating feature between BDD and BIID.

An essential strength of this work is that it is the first to study mental images with respect to BIID and BDD. Several assumptions that were previously only suspected were supported in the present study. Mental images emerge even in healthy control subjects (14\%), but they are rare compared to the two conditions (BDD: 74\%; BIID: 68\%). Sorene and colleagues (2006) hypothesised that the mental images of individuals with BIID relate to their desired body and are emotionally positive. In our study (in line with Sorene et al.), the contents of the images of BIID subjects clearly illustrate a presentation of the desired disability, which is associated with more positive emotions. We assume that mental imagery in BIID serves a very different function: Parallel to real, everyday life, intentionally evoked mental images may often run as a permanent mental movie in the brain, which shows life as a person with the desired disability. In contrast, mental imagery in BDD might have the function of not having to cope with stress or anxiety, getting attention or not having to work; images are unintentional, negative, and often overwhelming.

There were several limitations to the present study. First, the groups differed significantly on sociodemographic characteristics. Although there is no evidence in the literature that, for example, gender or age have an influence on mental images, it is possible that young women experience significantly 
more body-related mental images than older men. Second, the small sample size of the BDD and BIID groups limits the conclusions that can be made from this study, particularly for the mental imagery research questions. It remains unclear whether there is truly no difference, or whether effects are not sufficiently large to be detected in the present study. Moderate effects may still be clinically relevant. However, in small sample sizes, effects may also be overestimated (i.e., come out larger than they actually are; Friston, 2012). To support our significant results by post-hoc power analyses, we used the program $\mathrm{G}^{\star}$ Power 3.1.9.2 (Faul, Erdfelder, Lang \& Buchner, 2007). For the chi-squared tests (occurence of a mental image; concern/distress), power was about $96 \%$ ( $\beta$ error was not greater than $5 \%$ ). However, for sexual arousal, power was only $61 \%$ ( $\beta$ error $=39 \%)$, indicating the observed effects may be biased by the small sample. Finally, the chosen $p$ values could be criticised due to the large number of comparisons.

Future research should therefore replicate the present study with a greater number of participants. However, this may be difficult because most studies including BIID participants involve small sample sizes $(<20)$. Finally, the modified German version of the mental images interview has not yet been validated. The results clearly indicate that mental images play an important role in both BDD and in BIID. Although they also occur in healthy controls, they are much more frequent in both clinical groups. It seems likely that not only mental disorders, but also other parameters such as sociodemographic characteristics may have an effect on the formation of mental images. It may be necessary to specifically target for the positive mental images related to the desired handicap in BIID to improve treatment outcome in this group.

Declaration of Interest. The first and the last author are the clinical and the scientific director of the university-based outpatient unit for body dysmorphic disorder and related disorders.

\section{References}

American Psychiatric Association (APA). (1987). Diagnostic and statistical manual of mental disorders (3rd ed., rev.). Washington, DC: Author.

American Psychiatric Association (APA). (1994). Diagnostic and statistical manual of mental disorders (4th ed.). Washington, DC: Author.

American Psychiatric Association (APA). (2000). Diagnostic and statistical manual of mental disorders (4th ed., text rev.). Washington, DC: Author.

American Psychiatric Association (APA). (2013). Diagnostic and statistical manual of mental disorders (5th ed.). Washington, DC: Author.

Bortz J and Lienert GA (2008). Clipped statistics for clinical research. Heidelberg, Germany: Springer.

Brewin CR, Gregory JD, Lipton M and Burgess N (2010). Intrusive images in psychological disorders: Characteristics, neural mechanisms, and treatment implications. Psychological Review 117, 210-232. doi:10.1037/a0018113

Brown TA, Barlow DH and di Nardo PA (1994). Anxiety Disorders Interview Schedule for DSM-IV (ADIS-IV): Client Interview Schedule. Oxford: University Press.

Buhlmann U, Glaesmer H, Mewes R, Fama JM, Wilhelm S, Brähler E and Rief W (2010). Updates on the prevalence of body dysmorphic disorder: A population-based survey. Psychiatry Research 178, 171-175. doi:10.1016/j.psychres. 2009.05.002

Faul F, Erdfelder E, Lang A-G and Buchner A (2007). G*Power 3: A flexible statistical power analysis program for the social, behavioral, and biomedical sciences. Behavior Research Methods 39, 175-191. doi:10.3758/BF03193146

First MB (2005). Desire for amputation of a limb: Paraphilia, psychosis, or new type of identity disorder. Psychological Medicine 35, 919-928. doi:10.1017/S0033291704003320

First MB and Fisher CE (2012). Body integrity identity disorder: The persistent desire to acquire a physical disability. Psychopathology 45, 3-14. doi:10.1159/000330503

Friston K (2012). Ten ironic rules for non-statistical reviewers. Neuroimage 61, 1300-1310. doi:10.1016/ j.neuroimage.2012.04.018

Giummarra MJ, Bradshaw JL, Hilti LM, Nicholls ME and Brugger P (2012). Paralyzed by desire: A new type of body integrity identity disorder. Cognitive and Behavioral Neurology 25, 34-41. doi:10.1097/WNN.0b013e318249865a

Hackmann A, Bennet-Levy J and Holmes EA (2012). Techniques of imagination in cognitive behavior therapy. Weinheim, Germany: Beltz.

Hackmann A, Clark DM and McManus F (2000). Recurrent images and early memories in social phobia. Behaviour Research and Therapy 38, 601-610. doi:10.1016/S0005-7967(99)00161-8 
Hackmann A, Surawy C and Clark DM (1998). Seeing yourself through others' eyes: A study of spontaneously occurring images in social phobia. Behavioural and Cognitive Psychotherapy 26, 3-12.

Hirsch CR, Clark DM, Mathews A and Williams R (2003). Self-images play a casual role in social phobia. Behaviour Research and Therapy 41, 909-921. doi:10.1016/S0005-7967(02)00103-1

Holmes EA, Arntz A and Smucker MR (2007a). Imagery rescripting in cognitive behaviour therapy: Images, treatment techniques and outcomes. Journal of Behavior Therapy and Experimental Psychiatry 38, 297-305. doi:10.1016/ j.jbtep.2007.10.007

Holmes EA, Crane C, Fennell MJV and Williams JMG (2007b). Imagery about suicide in depression - 'Flash-forwards?'. Journal of Behavior Therapy and Experimental Psychiatry 38, 423-434. doi:10.1016/j.jbtep.2007.10.004

Holmes EA and Mathews A (2010). Mental imagery in emotion and emotional disorders. Clinical Psychology Review 30, 349-362. doi:10.1016/j.cpr.2010.01.001

Horowitz MJ (1967). Visual imagery and cognitive organization. Archives of General Psychiatry 123, $938-946$.

Horowitz MJ (1970). Image formation and cognition. New York, NY: Appleton Century Crofts.

Kasten E (2009). Body integrity identity disorder (BIID): Interrogation of patients and theories for explanation. Fortschritte der Neurologie-Psychiatrie 77, 16-24. doi:10.1055/s-0028-1100837

Kosslyn SM, Ganis G and Thompson WL (2001). Neural foundations of imagery. Neuroscience 2, 635-642. doi:10.1038/ 35090055

Margraf J (1994). Mini-Dips. Diagnostic Short Interview of Mental Disorders. Berlin, Germany: Springer.

Margraf J, Schneider S and Ehlers A (1991). Diagnostic Interview of Mental Disorders (DIPS). Berlin, Germany: Springer.

Neziroglu F, Khemlani-Patel S and Veale D (2008). Social learning theory and cognitive behavioral models of body dysmorphic disorder. Body Image 5, 28-38. doi:10.1016/j.bodyim.2008.01.002

Obernolte C, Schnell T and Kasten E (2015). The role of specific experiences in childhood and youth in the development of body integrity identity disorder (BIID). American Journal of Applied Psychology 4, 1-8. doi:10.11648/j.ajap.20150401.11

Onden Lim M (2013). Intrusive imagery in body dysmorphic disorder (Doctoral dissertation). Retrieved from http://www.unsworks.unsw.edu.au/primo_library/libweb/action/dlDisplay.do?vid=UNSWORKS\&docId=unsworks_11165

Oddo S, Möller J and Stirn A (2014). Body integrity identity disorder (BIID). In Stirn A, Stark R, Tabbert K, WehrumOsinsky S, \& Oddo S (Eds.), Sexuality, body and neurobiology. Basics and clinical pictures with focus on interdisciplinary (pp. 302-311). Stuttgart, Germany: Kohlhammer.

Osman S, Cooper M, Hackmann M and Veale D (2004). Imagery in people with body dysmorphic disorder: A study of its characteristics, and links to early beliefs and memories. Memory 12, 428-436.

Phillips KA (1986). The broken mirror. Understanding and treating body dysmorphic disorder. New York, NY: Oxford University Press.

Phillips KA (2004). Psychosis in body dysmorphic disorder. Journal of Psychiatric Research 38, 63-72. doi:10.1016/ S0022-3956(03)00098-0

Rief W, Buhlmann U, Wilhelm S, Borkenhagen A and Brähler E (2006). The prevalence of body dysmorphic disorder: A population-based survey. Psychological Medicine 36, 877-885. doi:10.1017/S0033291706007264

Ritter V and Stangier U (2016). Seeing in the mind's eye: Imagery rescripting for patients with body dysmorphic disorder. A single case series. Journal of Behavior Therapy and Experimental Psychiatry 50, 187-195. doi:10.1016/j.jbtep.2015.07.007

Rösner M (2014). Mental images in body dysmorphic disorder and in social anxiety disorder (Unpublished master's thesis). Braunschweig University, Braunschweig.

Sedda A and Bottini G (2014). Apotemnophilia, body integrity identity disorder or xenomelia? Psychiatric and neurologic etiologies face each other. Neuropsychiatric Disease and Treatment 10, 1255-1265. doi:10.2147/NDT.S53385

Sorene ED, Heras-Palou C and Burke FD (2006). Self-amputation of a healthy hand: A case of body integrity identity disorder. Journal of Hand Surgery (British and European Volume) 31, 593-595. doi:10.1016/J.JHSB.2006.05.022

Stangier U, Hungerbühler R and Meyer A (1996). German translation of the Body Dysmorphic Disorder Diagnostic Module (BDDDM) by K.A. Phillips. Unpublished manuscript, Department of Psychology, University of Frankfurt, Germany.

Stirn A, Thiel A and Oddo S (2010). Body integrity identity disorder (BIID). Clinical picture, diagnostics, therapy approaches. Weinheim, Germany: Beltz.

Veale D (2004). Advances in a cognitive behavioural model of body dysmorphic disorder. Body Image 1, 113-125. doi:10.1016/S1740-1445(03)00009-3

Veale D and Neziroglu F (2010). Body dysmorphic disorder. A treatment manual. Chichester, UK: Wiley-Blackwell.

Wild J, Hackmann A and Clark DM (2008). Rescripting early memories linked to negative images in social phobia: A pilot study. Behavior Therapy 39, 47-56. doi:10.1016/j.beth.2007.04.003

Willson R, Veale D and Freeston M (2016). Imagery rescripting for body dysmorphic disorder: A multiple-baseline singlecase experimental design. Behavior Therapy 47, 248-261. doi:10.1016/j.beth.2015.08.006

Cite this article: Grocholewski A, Bücker C, Kasten E, Osen B, Heinrichs N (2018). Mental Images in Body Integrity Identity Disorder (BIID) and in Body Dysmorphic Disorder (BDD): An Exploratory Study. Behaviour Change 35, 174-184. https:// doi.org/10.1017/bec.2018.17 